\title{
Reninoma: Uma Causa Rara de Hipertensão Endócrina
}

\section{Reninoma: A Rare Cause of Endocrine Hypertension}

Bernardo MARQUES $₫ 1$, Joana COUTO¹, Manuel C. LEMOS², Fernando RODRIGUES ${ }^{1}$

Acta Med Port 2020 Dec;33(12):835-838 - https://doi.org/10.20344/amp.11660

RESUMO

Os tumores justa-glomerulares são causas raras de hipertensão arterial de difícil controlo e cursam habitualmente com hipocaliémia, hiperreninémia e hiperaldosteronismo secundário. Descreve-se o caso de uma doente, de 45 anos, com história pessoal de hipertensão arterial de difícil controlo associada a hipocaliémia desde os 35 anos, medicada com quatro classes de anti-hipertensores. Do estudo realizado destaca-se hiperaldosteronismo secundário [aldosterona 44,3 ng/dL (4 - $28 \mathrm{ng} / \mathrm{dL}$ ), renina > $1000 \mathrm{mUl} / \mathrm{mL}(4,4$ - 46,16 mUl/ $\mathrm{mL}$ )] e tomografia axial computorizada, que identificou formação nodular heterogénea localizada no terço médio do rim direito, com $3,7 \mathrm{~cm}$ de diâmetro. Foi realizada nefrectomia parcial, cuja análise histológica confirmou o diagnóstico de reninoma. Após a cirurgia, verificou-se normalização dos doseamentos hormonais (aldosterona 9,2 ng/dL; renina 1,20 mUl/mL) e da pressão arterial. Pretende-se chamar a atenção para esta causa potencialmente curável de hipertensão arterial endócrina. A ressecção cirúrgica é o tratamento de escolha e leva à normalização da pressão arterial.

Palavras-chave: Aldosterona; Hipertensão/etiologia; Neoplasias do Rim; Renina; Sistema Justaglomerular

\section{ABSTRACT}

Juxtaglomerular tumours are rare causes of secondary hypertension. They typically present with difficult-to-manage hypertension, hypokalemia, hyperreninemia and secondary hyperaldosteronism. The authors describe a clinical case of a 45 years old female patient, with personal history of difficult-to-manage hypertension and hypokalemia since age 35 , medicated with four types of anti-hypertensive agents. An analytical study was performed, which revealed secondary hyperaldosteronism [aldosterone $44.3 \mathrm{ng} / \mathrm{dL}(4-28 \mathrm{ng} / \mathrm{dL}$ ), renin $>1000 \mathrm{mIU} / \mathrm{mL}(4.4-46.2 \mathrm{mIU} / \mathrm{mL})]$. Abdominal computed tomography scan identified a heterogeneous nodule located in the middle third of the right kidney, with $3.7 \mathrm{~cm}$. Partial nephrectomy was performed and histological analysis confirmed the diagnosis of reninoma. After surgery, the patient had normal levels of aldosterone $(9.2 \mathrm{ng} / \mathrm{dL})$ and renin $(1.20 \mathrm{mlU} / \mathrm{mL})$, as well as normal blood pressure. The authors want to highlight this potentially curable cause of endocrine hypertension. Surgical resection is the treatment of choice and leads to normalization of blood pressure.

Keywords: Aldosterone; Hypertension/etiology; Juxtaglomerular Apparatus; Kidney Neoplasms; Renin

\section{INTRODUÇÃO}

A hipertensão arterial (HTA) é um fator de risco preponderante para as doenças cardiovasculares, que se encontram atualmente entre as mais importantes causas de morbilidade e mortalidade $1^{1,2} \mathrm{~A}$ maioria dos casos consideram-se idiopáticos, embora cerca de $15 \%$ sejam de causa secundária. A incidência desta entidade é superior na idade pediátrica, em que $50 \%$ dos casos de HTA são de causa secundária, nomeadamente doença parenquimatosa renal e endócrina. ${ }^{1-3}$

A HTA pode ser a manifestação inicial de pelo menos nove patologias endócrinas (Tabela 1). Este diagnóstico deve ser considerado em doentes jovens ou com HTA de difícil controlo. Atendendo à possibilidade de realização de cirurgia com intuito curativo ou otimização da terapêutica médica dirigida, é importante a identificação da causa endócrina da HTA. ${ }^{3}$

O tumor justa-glomerular ou reninoma é uma causa rara de HTA endócrina e apresenta-se mais frequentemente em adolescentes ou adultos jovens. Estes tumores cursam habitualmente com HTA de difícil controlo, hipocaliémia e hiperaldosteronismo secundários à hipersecreção de renina pelo tumor. $^{4}$

\section{CASO CLÍNICO}

Mulher de 45 anos, com história pessoal de HTA de difícil controlo com 10 anos de evolução, associada a hipocaliémia, com necessidade de correção. Encontrava-se medicada com perindopril/amlodipina $10 / 5 \mathrm{mg}$, metoprolol $100 \mathrm{mg}$ e espironolactona $50 \mathrm{mg}$, com razoável controlo tensional.

Foi observada em consulta pelo seu médico assistente, tendo realizado análises sob a sua medicação anti-hipertensora habitual, que revelaram: aldosterona $>132 \mathrm{ng} / \mathrm{dL}$ $(3,7-43,2 \mathrm{ng} / \mathrm{dL})$, renina $>550 \mathrm{mUl} / \mathrm{mL}(4,2-59,7 \mathrm{mUl} /$ $\mathrm{mL}$ ); ureia, creatinina, função tiroideia, metanefrina e normetanefrina urinárias normais. Realizou ecocardiograma que não revelou sinais de cardiopatia hipertensiva e tomografia axial computorizada abdominal, que revelou uma formação nodular heterogénea no terço médio do rim direito, com $3,7 \mathrm{~cm}$ de diâmetro (Fig. 1).

Por suspeita de hipertensão endócrina foi encaminhada para uma consulta de Endocrinologia.

Na primeira consulta, objetivou-se tensão arterial (TA) de 152/102 mmHg. Repetiu análises sem suspensão da medicação anti-hipertensora, verificando-se concentrações plasmáticas de aldosterona de $44,3 \mathrm{ng} / \mathrm{dL}$ (4 - $28 \mathrm{ng} / \mathrm{dL}$ ); renina $>1000 \mathrm{mUI} / \mathrm{L}(4,4-46,1 \mathrm{mUI} / \mathrm{L})$ e potássio de 3,3

\footnotetext{
1. Serviço de Endocrinologia. Instituto Português de Oncologia de Coimbra Francisco Gentil, EPE. Coimbra. Portugal.

2. CICS-UBI, Centro de Investigação em Ciências da Saúde. Universidade da Beira Interior. Covilhã. Portugal.

$\triangle$ Autor correspondente: Bernardo Marques. ber.marques89@gmail.com

Recebido: 10 de dezembro de 2018 - Aceite: 08 de maio de 2019 | Copyright @ Ordem dos Médicos 2020
} 
Tabela 1 - Causas de hipertensão arterial endócrina

Feocromocitoma/paraganglioma

Hiperplasia congénita da suprarrenal

Síndrome de Cushing

Hiperparatiroidismo

Acromegalia

Hiperaldosteronismo primário

Hipertiroidismo

Hipotiroidismo

Reninoma

(3,5 - 4,5 mmol/L). Após correção da hipocaliémia com cloreto de potássio e suspensão de espironolactona durante quatro semanas, realizou novo estudo analítico, que revelou aldosterona $13,6 \mathrm{ng} / \mathrm{dL}$ e renina $1145,5 \mathrm{mUI} / \mathrm{mL}$.

Foi colocada a hipótese de reninoma e realizou-se nefrectomia parcial direita, com excisão de tumor com $2,5 \mathrm{~cm}$. O exame histológico revelou formação tumoral constituída por células poligonais, com citoplasma abundante eosinofílico. O estudo imunohistoquímico revelou positividade para vimentina e CD34. Os aspetos observados eram compatíveis com reninoma (Fig. 2).

No período pós-operatório verificou-se normalização da TA (Tabela 2). Realizou-se avaliação analítica três meses após a cirurgia, com remissão do hiperaldosteronismo e hiperreninémia (aldosterona 9,2 $\mathrm{ng} / \mathrm{dL}$ e renina 1,20 mUl/ $\mathrm{mL}$ ). Realizou também monitorização ambulatória da TA, que confirmou a sua normalização e ecografia renal, que confirmou a cura cirúrgica. Mantém-se normotensa e com doseamentos hormonais normais, dois anos após a cirurgia.

\section{DISCUSSÃO}

Os reninomas são tumores raros (descritos cerca de 100 casos), constituem uma causa potencialmente curável de HTA secundária e apresentam geralmente comportamento benigno (encontra-se descrito um caso de reninoma metastizado). ${ }^{5} \mathrm{O}$ seu diagnóstico deve ser considerado em doentes jovens com HTA de difícil controlo, hiperaldosteronismo, hiperreninémia e hipocaliémia. A hipersecreção de renina pelo tumor induz a ativação do angiotensinogénio em angiotensina I e posteriormente em angiotensina II. Este é um importante vasoconstritor e estimula a secreção

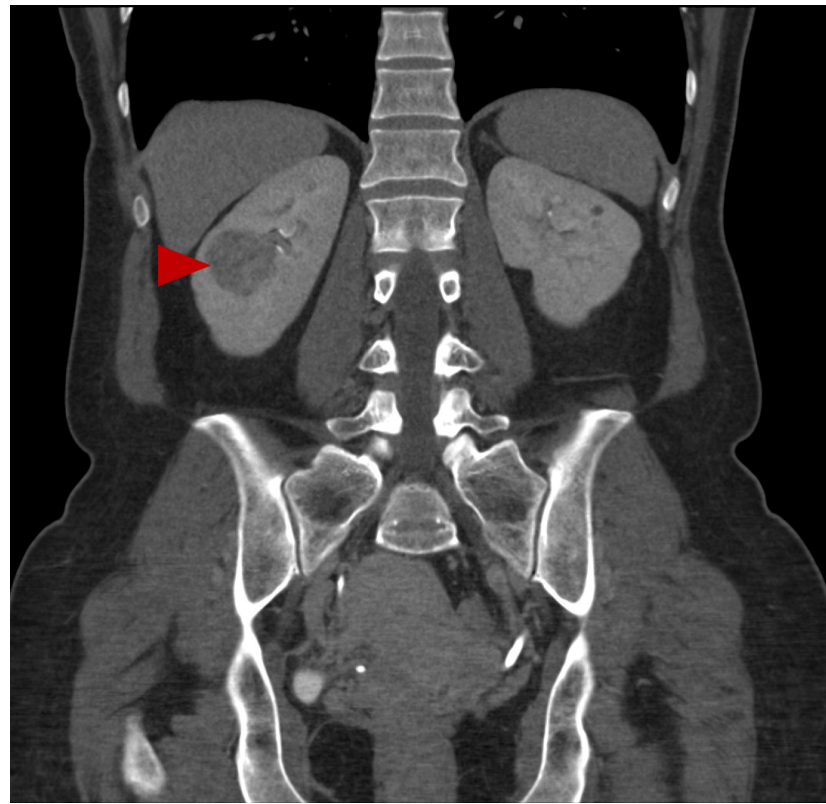

Figura 1 - TC abdominal demonstrando nódulo heterogéneo no terço médio do rim direito, com 3,7 cm (seta)

de aldosterona pelo córtex da suprarrenal. A excessiva ativação do eixo renina-angiotensina-aldosterona é responsável pelo desenvolvimento de HTA, hipocaliémia e hiperaldosteronismo secundário.

As lesões de órgão-alvo associadas ao reninoma são relativamente frequentes, nomeadamente a retinopatia hipertensiva ligeira ou moderada (24\%). Os casos de edema da papila são raros (6\%) e associam-se, na maioria dos casos, a défices no campo visual ou diminuição da acuidade visual, que não foram descritos pela doente. ${ }^{6}$

Foi descrita proteinúria e insuficiência renal em cerca de $11 \%$ e $3 \%$ dos doentes, respetivamente. A cardiopatia hipertensiva foi excluída nesta doente, mas é documentada em cerca de $7 \%$ dos casos. Tal como nas lesões de órgão-alvo associadas a outros casos de HTA, o diagnóstico precoce e adequado controlo tensional são fundamentais para a sua prevenção. ${ }^{6}$

Dong et $a{ }^{4}$ propuseram uma classificação dos reninomas em três categorias, baseada numa série de 71 casos: a variante típica, que representa a maioria dos reninomas, com hiperreninémia, hiperaldosteronismo, hipocaliémia e HTA; a variante atípica, com HTA e normocaliémia e uma variante não funcional, caracterizada por TA normal e

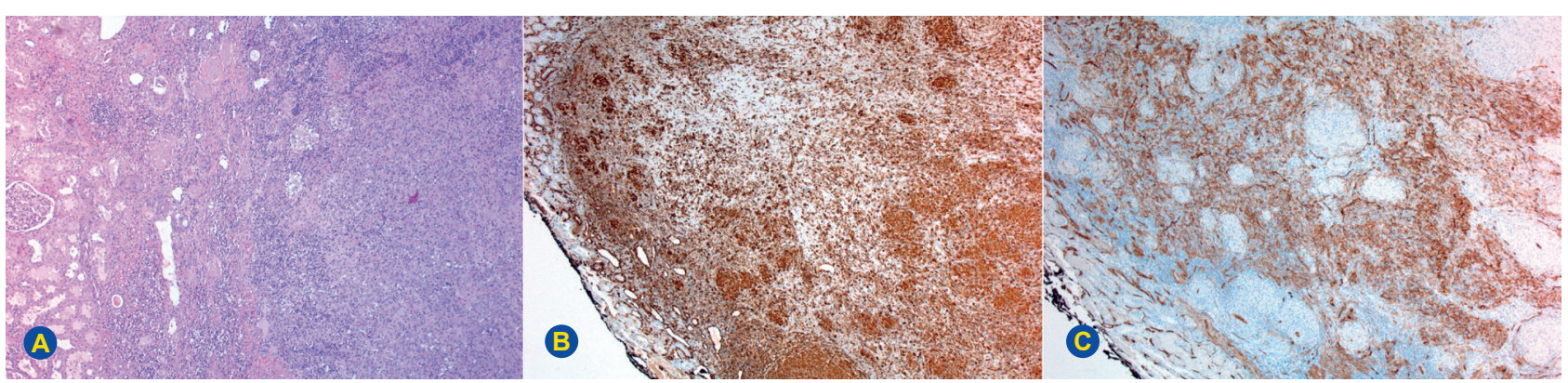

Figura 2 - Imagem histológica com interface nefrónio e reninoma, com citoplasma abundante eosinofílico (A). O estudo imunohistoquímico revelou positividade difusa para vimentina (B) e focal para CD34 (C). Todas as imagens apresentam ampliação 5x. 
normocaliémia. Este caso apresentou-se com os quatro parâmetros, pelo que se pode classificar como variante típica.

Em caso de suspeita de HTA secundária, deve-se proceder a estudo analítico, que deve incluir ionograma, cálcio, função tiroideia, metanefrina plasmática, aldosterona e renina. Nestes casos, a doença renovascular ou parenquimatosa deve ser também excluída, pois constituem a principal causa de HTA nos jovens. ${ }^{3}$

Idealmente, os doseamentos hormonais devem ser realizados sem restrição de sal e com caliémia normal. Os antagonistas do recetor dos mineralocorticóides devem ser suspensos durante pelo menos 4 semanas, pois constituem a classe de fármacos que mais interfere no doseamento da renina e aldosterona. Os resultados poderão ser interpretados mantendo os restantes fármacos anti-hipertensores, caso a suspensão ou substituição dos mesmos não possa ser realizada em ambiente controlado, evitando assim o atraso no diagnóstico. ${ }^{3}$ No estudo deste caso, atendendo à indicação cirúrgica independentemente do seu comportamento funcional, à elevada suspeita clínica e para maior segurança da doente, optou-se por não suspender os fármacos anti-hipertensores para a realização dos doseamentos hormonais, à exceção da espironolactona.

No estudo imagiológico apresentam-se geralmente como massas heterogéneas de tamanho variável, de difícil distinção, por vezes, do carcinoma de células renais (CCR). A ressonância magnética nuclear tem particular interesse, já que apresentará maior acuidade diagnóstica na distinção entre estes dois tumores. ${ }^{7,8}$

O CCR cursa frequentemente com HTA, pelo que o cateterismo das veias renais poderá ser necessário para confirmar o diagnóstico. No entanto, este é um procedimento invasivo, com elevada taxa de falsos negativos, justificado pela natureza cortical e drenagem venosa pericapsular da maioria dos reninomas descritos. , $10^{10}$

O diagnóstico histológico também é desafiante, atendendo ao escasso número de casos publicados, sendo confirmado pela positividade da renina no citoplasma, embora esta positividade possa também ser observada no CCR, nefroblastoma, ou oncocitoma renal. O estudo imunohis- toquímico é essencial para excluir os diagnósticos acima referidos, sendo que, tal como neste caso, a maioria das células neoplásicas de reninoma são difusamente imunorreativas à vimentina e ao CD34. ${ }^{11,12}$

A ressecção cirúrgica do tumor, com nefrectomia parcial, é o tratamento de escolha e leva à normalização da TA. $4,6,9,13$

Atendendo à raridade desta entidade, não há atualmente evidência que suporte recomendações específicas para o seguimento destes doentes. Em todos os casos reportados é sugerida vigilância clínica e laboratorial após a cirurgia.4,6,9,13 Neste caso, o tumor não apresentava características histológicas sugestivas de risco elevado de recorrência, nomeadamente invasão vascular ou necrose tumoral, pelo que se justificará apenas seguimento clínico e analítico. ${ }^{5} \mathrm{Em}$ caso de suspeita de doença recorrente, deverá ser solicitado estudo imagiológico.

\section{PROTECÇÃO DE PESSOAS E ANIMAIS}

Os autores declaram que os procedimentos seguidos estavam de acordo com os regulamentos estabelecidos pelos responsáveis da Comissão de Investigação Clínica e Ética e de acordo com a Declaração de Helsínquia da Associação Médica Mundial.

\section{CONFIDENCIALIDADE DOS DADOS}

Os autores declaram ter seguido os protocolos do seu centro de trabalho acerca da publicação de dados.

\section{CONSENTIMENTO DO DOENTE}

Obtido.

\section{CONFLITOS DE INTERESSE}

Os autores declaram não ter conflitos de interesses relacionados com o presente trabalho.

\section{FONTES DE FINANCIAMENTO}

Este trabalho não recebeu qualquer tipo de suporte financeiro de nenhuma entidade no domínio público ou privado.

\section{REFERÊNCIAS}

1. Whelton PK, Carey RM, Aronow WS, Casey DE Jr, Collins KJ, Dennison Himmelfarb C, et al. 2017ACC/AHA/AAPA/ABC/ACPM/AGS/APhA/ ASH/ASPC/ NMA/PCNA Guideline for the Prevention, Detection, Evaluation, and Management of High Blood Pressure in Adults: Executive Summary: A Report of the American College of Cardiology/ American Heart Association Task Force on Clinical Practice Guidelines. Hypertension. 2018;71:1269-324.

2. Mills KT, Bundy JD, Kelly TN, Reed JE, Kearney PM, Reynolds KP, et al. Global disparities of hypertension prevalence and control: a systematic analysis of population-based studies from 90 countries. Circulation. 2016;134: 441-50.

3. Young WF, Calhoun DA, Lenders JW, Stowasser M, Textor SC. Screening for endocrine hypertension: an endocrine society scientific statement. Endocr Rev. 2017;38:103-22.

4. Dong D, Li H, Yan W, Xu W. Juxtaglomerular cell tumor of the kidney - a new classification scheme. Urol Oncol. 2010; 28:34-8.

5. Duan X, Bruneval P, Hammadeh R, Fresco R, Eble J, Clark JI, et al. Metastatic juxtaglomerular vell tumor in a 52-year-old man. Am J Surg Pathol. 2004;28:1098-102.
6. Wong L, Hsu TH, Perlroth MG, Hofmann LV, Haynes CM, Katznelson L. Reninoma: case report and literature review. J Hypertens. 2008; 26:36873

7. Suhai K, Aitao G, Haiyi W, Lu M, Zongyu X, Jinglong L, et al. Magnetic resonance imaging features of a juxtaglomerular cell tumor. J Clin Imaging Sci. 2015;5:68-76.

8. Faucon A, Bourillon C, Grataloup C, Baron S, Bernadet-Monrozies P Vidal-Petiot $E$, et al. Usefulness of magnetic resonance imaging in the diagnosis of juxtaglomerular cell tumors: a report of 10 cases and review of the literature. Am J Kidney Dis. 2019;73:566-71.

9. Trnka P, Orellana L, Walsh M, Pool L, Borzi P. Reninoma: an uncommon cause of renin-mediated hypertension. Front Pediatr. 2014;2:89-97.

10. Wolley M, Gordon RD, Stowasser M. Reninoma: the Importance of renal vein renin ratios for lateralisation and diagnosis. Am J Nephrol. 2014;39:16-9.

11. Tanaka T, Okumura A, Mori H. Juxtaglomerular cell tumor. Arch Pathol Lab Med. 1993;117:1161-4.

12. Kuroda N, Gotoda H, Ohe C, Mikami S, Inoue K, Nagashima Y, et al. Review of juxtaglomerular cell tumor with focus on pathobiological 
aspect. Diagn Pathol. 2011;6:80-6.

13. Torricelli FC, Marchini GS, Colombo JR, Coelho RF, Nahas WC,

Srougi M. Nephron-sparing surgery for treatment of reninoma: a rare renin secreting tumor causing secondary hypertension. Int Braz J Urol. 2015;41:172-6.

An Unusual Case of Invasive Encapsulated Papillary

Carcinoma of the Breast: A Case Report

Um Caso Raro de Carcinoma Papilar Encapsulado

Invasivo da Mama: Um Caso Clínico

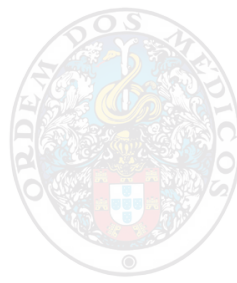

Xavier de SOUSAR**1. Pedro Santos FERREIRA**1. Isabel Lopes MARTINS1. Manuel Vitor RIGUEIRA

Acta Iled Port 2020 Dec;33(12):838-842

ABSTRACT

Papillary carcinoma of the breast is a rare type of breast malignancy, making up less than $2 \%$ of breast cancers, and only some are encapsulated papillary carcinoma. Usually, EPC are low-grade luminal like tumors. The authors report a case of a 50-year-old female with the diagnosis of an unusual high-grade triple negative encapsulated papillary carcinoma with an area of invasive papillary carcinoma and negative axillary lymph nodes. Considering its rarity, the description of this case and its clinical management are important in order to better understand this entity and choose the best clinical approach.

Keywords: Breast Neoplasms; Carcinoma, Papillary; Lymph Nodes

RESUMO

o carcinoma papilar é um tipo raro de cancro da mama, com incidência inferior a 2\% sendo anenas uma parte carcinoma papila encapsulado. Normalmente, o carcinoma papilar encapsulado apresenta-se como um carcinoma da mama tipo luminal e bem diferen. ciado. Os autores apresentam o caso de una mulher de 50 anos com o diagnóstico de carcinoma papilar encapsulado triplo negativa e pouco diferenciado com uma área de carcinoma papilar invasivo e gânglios linfáticos axilares negativos. Considerando a sua rariJade, a descrição deste caso e da sua abordagem torna-se importante um melhor entendimento desta entidade e para compreender a melhor prática clínica a utilizar.

Palavras-chave: Carcinoma Papilar; Neoplasias da Mama; Nódulos Linfáticos

\section{INTRODUCTION}

Malignant papillary breast lesions are a rare type o: breast cancer and are presented as a heterogeneous group of lesions with different histological types, such as, ducta carcinoma in situ arising in an intraductal papilloma, papil. lary ductal carcinoma in situ, encapsulated papillary carcinoma (EPC), solid and invasive papillary carcinoma (IPC), occurring mostly during the fifth and sixth decades of life. EPC, originally considered as an in situ carcinoma, differentiated by the presence of a fibrous capsule and absence of a myoepithelial cell laver, is now seen as an indolent and low-grade invasive carcinoma which rarely metastasizes to axillary lymph nodes ${ }^{1-3}$ without evidence of invasion. ${ }^{4}$ Hormone receptors lestrogen and progesterone receptors (ER PR) are usually positive and HER2 negative. ${ }^{1}$ EPC may rarely be associated with invasive carcinoma when the carcinoma extends beyond the fibrous layer. ${ }^{3}$ The authors only encountered four articles reporting high-grade, triple negative EPC with invasive component

\section{CASE REPORT}

A 50-year-old female, previously healthy and with no family history of breast cancer, was referred to a breast surgery clinic for presenting a painless palpable mass on the outer-lower quadrant of the left breast with a month of evoIution

Breast ultrasound and mammography showed a lobulated nodular image of $3.3 \times 2 \mathrm{~cm}$, (BIRADS 4A). Core needle biopsy (CNB) was performed and demonstrated small fragments of fibroadipose tissue and scarce breast parenchyma

A 6-month follow-up left breast ultrasound and mammography showed a cystic mass with a solid component of $9.1 \times 5.4 \mathrm{~cm}$ (BIRADS 4A) (Fig. 1). Fine needle aspiration cytology was performed, which was negative for cancer cells

Breast MRI was performed three months later, which showed a voluminous cystic lesion of $12.7 \times 10.5 \mathrm{~cm}$ associated with a solid component and skin thickening on the left breast (BIRADS 4A) (Fig. 2).

Given the growth and volume of the breast lesion, which was discordant with previous histological and cytological findings, an excisional biopsy was performed to clarify the lesion's nature (Fig. 3) (Fig. 4). The histopathological report of the excisional biopsy revealed EPC with $11 \mathrm{~cm}$ of diameter associated with an invasive component of $5 \mathrm{~cm}$, with tumor present in the surgical margins. Tumor cells were 